\title{
Precision oncology in patients with nonmetastatic disease: emerging reality or illusion
}

\author{
Persefoni Antoniou ${ }^{1,2}$, Demosthenes E Ziogas ${ }^{1,3}$, Michail Mitsis ${ }^{2,4}$ \& Dimitrios H \\ Roukos*, 1,2,4,5 \\ ${ }^{1}$ Centre for Biosystems \& Genome Network Medicine, loannina University, loannina, Greece \\ ${ }^{2}$ Department of Surgery, loannina University Hospital, loannina, Greece \\ ${ }^{3}$ Department of Surgery, 'G Hatzikosta' General Hospital, loannina, Greece \\ ${ }^{4}$ Cancer Biobank Centre, University of loannina, loannina, Greece \\ ${ }^{5}$ Department of Systems Biology, Biomedical Research Foundation of the Academy of Athens (BRFAA), Athens, Greece \\ *Author for correspondence: Tel.: +30 265100 5572; droukos@uoi.gr
}

“'the definitive evidence on genetic, genomic and transcriptional heterogeneity guides emerging research to develop robust biomarkers and multiple targeted drugs"

First draft submitted: 9 March 2019; Accepted for publication: 8 April 2019; Published online:

7 June 2019

Keywords: biomarkers $\bullet$ breast cancer $\bullet$ genome $\bullet$ oncotargets $\bullet$ precision oncology $\bullet$ transcriptome

Despite advances in the standardization of surgical resection and adjuvant or neo-adjuvant treatment (NAT), therapeutic resistance and relapse rates remain alarmingly high [1]. Breakthrough genome sequencing and editing technologies [2,3] represent the most rational roadmap toward precision oncology [4-6]. The realization of this goal is just beginning with the development of robust static and dynamic biomarkers [7] as well as the discovery of new oncotargets as bases for targeted drug development [8]. This Commentary discusses the potential and the challenges to achieve the goal of individualized drug-response prediction and therapy.

Advances in surgery, radiotherapy, chemotherapy, targeted drugs as well as evidence-based strategy with randomized control trials and meta-analyses have improved oncological outcomes of patients with resectable or locally advanced disease (M0 stage). However, with the exception of rapid progress in a few cancer types including breast cancer, for most other major cancer types including lung, pancreatic and esophageal tumors progress is too slow [1].

The major problem explaining this modest improvement of survival rates of these tumors is the early resistance and relapse following adjuvant treatment or NAT [9]. The lack of robust prognostic and predictive biomarkers and the limited number of targeted drugs added to chemotherapy for this nonmetastatic setting explains the unmet need for overcoming intrinsic and acquired drug resistance.

These clinical challenges could be overcome by integrating next-generation sequencing technologies (NGS) for the identification of robust prognostic and predictive biomarkers, as well as the discovery of new oncotargets. Genome-based biomarkers could potentially include intratumor heterogeneity and circulating cell free DNA-NGS (cfDNA-NGS). The evidence of extensive genetic heterogeneity applying NGS can reveal druggable mutations as basis for the development of targeted drugs [10]. In this Commentary, the challenges and the potential to translate these genomic and transcriptomic discoveries into clinical oncology are discussed.

\section{Clinical standards: advances \& challenges}

Beyond standardization of multimodal treatment including surgery, radio- and chemotherapy of solid tumors, targeted drugs represent the major hope for improving oncological outcomes. Targeted therapy either alone or more often in combination with adjuvant or neo-adjuvant chemo-radiotherapy could substantially reduce relapse and improve survival. However, in resectable tumors without distant metastases (stage M0), a few only tumor-guided agents have been approved [1]. 


\section{Low, moderate $\&$ high aggressive cancer types}

Based on high quality evidence from randomized control trials regarding relapse-free survival and overall survival rates, we have classified tumors into low, moderate and highly aggressive cancer types. Breast and colorectal cancer belong on low aggressiveness tumors, considering their excellent survival rates in early stages of the disease. For instance, early resistance and relapse rates less than $10 \%$ have recently been reported with a combination of targeted drugs such as pertuzumab or renatinib added to standard trastuzumab plus chemotherapy for HER2positive resectable breast cancer $[11,12]$. Similarly, prognosis of colorectal cancer $(\mathrm{CRC})$ is very good in resectable nonmetastatic disease, while even in removable CRC liver metastases 5-year survival rates are reported to be over $30 \%[13]$.

In a series of moderate aggressiveness cancer types including gastric, melanoma and kidney, 5-year disease-free survival rate after surgical resection and adjuvant chemotherapy and/or targeted therapy range between 40 and 59\%, while for advanced and metastatic disease survival rates remain disappointing $[1,14,15]$. On the other hand, intrinsic resistance and relapse rates remain very high even in early tumor stages carcinomas, as for instance in pancreatic adenocarcinoma (PDA) with a 5 year disease-free survival and overall survival $18.6 \%$ and $30-40 \%$, respectively [16]. Similarly, for non-small-cell lung cancer the benefit of post-operative adjuvant therapy on overall survival was only $5 \%$ [17], suggesting the limited benefit of adjuvant systemic chemotherapy for this aggressive cancer type.

\section{Unmet needs}

Despite these advances mentioned above, multiple challenges remain, including late recurrence rates in HER2positive and triple-negative breast cancer, while for moderate and high aggressiveness tumors progress for locally advanced and metastatic disease is too slow. The validity and continuously reduced cost of NGS to identify interpatient and intra-patient genetic and genomic heterogeneity provide high expectations to overcome drug resistance, relapse and cancer related death rates $[2,4]$

\section{Cancer genome \& transcriptome analysis: published data \& progress}

With the completion of the draft of the first human genome sequencing in the beginning of this millennium and the first successful integration of NGS technologies into the Encyclopedia of DNA Elements ENCODE project $[2,18]$, substantial progress has been achieved in genome and transcriptome analysis. A substantial progress has been noted from the static single tumoral biopsy-NGS analysis [19] to spatiotemporal exploration of dynamic emergence of genomic subclones to identify intra-tumor heterogeneity [4] and serial cfDNA-NGS [20] to detect circulating genomic subclones (cGSs) as biomarkers of drug resistance and relapse [21]. These two strategies could provide important clinical implications including, the development of static and dynamic biomarkers as well as new oncotargets and targeted drugs which seems to be a realistic goal $[6,7]$.

\section{Single biopsy for NGS analysis}

Substantial progress with the identification of new cancer driver genes, mutations, oncotargets and molecular classification of diverse cancer types has already been observed in the post-NGS era of clinical samples after 2010. The evidence on extensive genetic heterogeneity has led to recommendation on large scale genome sequencing studies for the valid identification of new cancer driver genes and druggable mutations [19]. Based on this recommendation several studies have reported valid data. In the largest cancer-type specific whole exome sequencing (WES) study reported, Campbell et al. have identified seven novel significantly mutated genes and three new amplifications that could be translated into immunotherapy for adenocarcinoma and small-cell lung cancer [22].

In a large-scale whole genome sequencing (WGS) breast cancer study on 560 tumors, five novel cancer genes were found [23]. In a recent overview of WES or WGS with or without RNA-seq [10], over 100 novel cancer driver genes or recurrent mutated genes have been identified for many cancer types. Moreover two novel cancer therapeutic targets have been detected, including the BCAR4 in a WES-study for cervical tumors which was described for the first time for cancer in general. In addition, the well-known $H E R 3$ gene could represent a new therapeutic target for cervical cancer [24].

Genome analysis with static single biopsy whole exome, whole genome with or without RNA-seq have identified molecular classifications for several cancer types including renal, pancreatic and colorectal cancer. In a WES study on 663 renal tumors a precise molecular classification with diagnostic, prognostic and therapeutic relevance has been proposed [25]. 
Potential development of molecular classification with therapeutic implications on pancreatic adenocarcinoma could substantially improve current poor survival rates. Based on these urgent needs, Bailey et al. conducted genome and transcriptome analysis on patients to identify a new molecular classification [26]. They have analyzed 204 patients from whom 179 had WGS and 94 RNA-seq. This genomic and transcriptomic evaluation has revealed the molecular subtypes squamous; pancreatic progenitor; immunogenic; and aberrantly differentiated endocrine exocrine. Furthermore, in CRC a consensus on novel molecular transcriptomic subtypes has recently been reported. An international consortium has used 18 datasets with a total of 4151 patients in the effort to develop a new molecular classification. Multiple gene expression platforms have been applied for a novel CRC taxonomy microsatellite instability (MSI) immune which accounts for only $15 \%$ while the remaining $85 \%$ includes canonical, metabolic and mesenchymal subtypes. The mesenchymal subtype was associated with the worst relapse-free and overall survival rates. Among patients who have experienced relapse, there was a better prognosis in the CMS2 subgroup compared to the MSI subtype [27]. However, this consensus of molecular classification requires validation to the categorization of gene expression subtypes with further transcriptome analysis studies.

\section{Single liquid biopsy: noninvasive early diagnosis}

Noninvasive circulating cell-free DNA alterations are attracting major clinical interest as prognostic and predictive biomarkers for decision-making treatment and patient monitoring. These genomic mutations can be identified with cfDNA-NGS for cancer diagnosis, early detection and screening, prediction of therapeutic resistance and, probably, new therapeutic strategies [10].

Blood tests could be used for early diagnosis of resectable tumors. Two positive studies for noninvasive diagnosis, applying cfDNA-NGS have recently been reported. Cohen et al. have analyzed 1005 patients with eight different resectable cancer types and reported high sensitivity rates over $70 \%$. Based on this success they have termed this test as the CancerSeek test for these cancer types [28]. Another study applying KRAS in combination with four protein biomarkers on 221 PDA patients could increase the sensitivity rates to 64\% for this cancer type [29]. Shifting from these retrospective studies to emerging large scale cfDNA-NGS trials, evidence-based noninvasive diagnosis of resectable tumors could be integrated into clinical practice. The Circulating Cell-free Genome Atlas Study (CCGA; NCT02889978) has explored the clinical utility of circulating cell-free DNA to develop a blood test for early diagnosis [30]. More than 12,000 participants are using deep sequencing of circulating cell-free nucleic acids to develop assays to detect cancer early in blood. Even though high sensitivity has been reported for colorectal, ovarian, pancreatic, hepatocellular cancer, for other cancer types such as breast and lung low sensitivity rates have been reported, highlighting the need for more large-scale studies.

\section{Static \& dynamic genomics \& transcriptomics}

Applying multiregional NGS analysis from different geographic areas has unraveled intratumor heterogeneity (ITH) as an independent prognostic and predictive biomarker to guide precise individualized therapy [4]. In the largest static MR-NGS study on 100 patients with non-small-cell lung cancer and 327 tumor samples, extensive ITH was observed in a median of $30 \%$ of samples, with identification of druggable mutations in 17 patients [31]. Similarly, ITH was identified in 12 of 36 breast cancer patients in whom MR-NGS and targeted NGS was conducted [32].

Shifting from static to dynamic ITH, Yates et al. have evaluated the dynamic emergence of genomic clones before and after NAT of breast cancer. They have analyzed 50 breast cancer patients and 303 samples. In 13 patients druggable mutations were identified after NAT, which have emerged from dynamic clonal diversification [33]. These data suggest the emergence of new oncotargets in response to therapy.

Furthermore, two studies were conducted using targeted NGS and WES for multiple cancer types, with 265 and 86 patients respectively [34,35], identifying potential biomarkers and targetable mutations that can have a clinical impact. In colorectal cancer, ITH can be used as a prognostic factor helping in the discovery of early liver metastases, as shown in a study with 88 patients [36]. Several other smaller studies on static and dynamic emergence of ITH for breast, ovarian and colorectal cancer have reported important clinical implication of tumor heterogeneity, by identifying mutations and chromosomal rearrangements [8].

Beyond early diagnosis and potential screening, noninvasive serial cfDNA-NGS could be used as a biomarker for patient monitoring to predict acquired resistance and relapse several months before clinical and imaging diagnosis.

Such progress has been observed in several studies for metastatic solid tumors. For example, Frenel et al. analyzed 159 samples from patients with several metastatic cancer types during and after treatment [37], and in another study an additional 159 samples for metastatic colorectal cancer patients were acquired [38], showing that serial-cfDNA 
can be used as a prognostic and predictive biomarker. Smaller studies for prostate, breast and esophageal cancer have been conducted with a limited number of patients that further show its potential as a patient monitoring tool $[21,39]$.

\section{Limitations of genomic studies}

Despite highly promising data on the clinical utility of static and spatiotemporal genome and transcriptome analysis, multiple challenges remain unresolved. First, the vast majority of studies available on static genome analysis does not meet the recommendations by Lawrence et al. [19] for large-scale studies with significant difference $(\mathrm{p}<0.01)$ to assess valid discoveries on new cancer driver genes, oncotargets and molecular classifications. Second, available studies evaluating tumoral and circulating heterogeneity in time and space are small, heterogeneous and lacking a prospective protocol to draw conclusions. In contrast to this concept of spatiotemporal emergence of tumor heterogeneity, a few studies have found similarity between primary and metastatic tumors, supporting the model of pre-existing minor cell subpopulations within the primary tumor [10].

\section{Overcoming challenges \& conclusions}

High hopes for improving relapse and cancer-related death rates have risen by the validation for both ITH and cGSs of the primary tumor in appropriately designed genomic studies. In contrast to current the static tumor homogeneity-based approach with single biopsy, multiregional tumoral and matched serial liquid biopsies open new diagnostic, predictive and therapeutic avenues. Revealing the intra-individual tumoral and circulating mutational landscape by comparing ITH with cGSs could not only improve our understanding on molecular mechanisms underlying individualized drug resistance and relapse, but also to overcome current unmet research and clinical needs.

\section{New generation of spatiotemporal patient-centric genomic trials}

For an evidence-based strategy, a new design of clinicogenomic trials is required to validate the clinical utility of genomic and transcriptomic analysis. These trials should include a complete set of clinical, histopathological, Classification of Malignant Tumors Tumor, Node, Metastasis (TNM) staging, imaging data as well as treatment and follow-up events including relapse or death for each individual patient. Tumoral and multiple patient-derived samples from different areas of the primary tumor as well as liquid biopsies before, during and after treatment are obtained for a subsequent genomic and transcriptomic analysis. These clinicogenomic analyses should be conducted in large-scale studies, considering the extensive genetic, genomic and transcriptional heterogeneity. Identification and validation of dynamic ITH and serial cGSs detection in these clinicogenomic trials could establish tumoral and circulating, genetic and genomic heterogeneity as crucial prognostic and predictive biomarkers to improve both primary and secondary decision-making treatment to reduce intrinsic and acquired drug resistance and relapse rates [10].

In summary, the definitive evidence on genetic, genomic and transcriptional heterogeneity guides emerging research to develop robust biomarkers and multiple targeted drugs to successfully treat intratumor and circulating clonal variability.

Although single biopsy NGS studies have identified multiple new cancer driver genes, oncotargets and molecular classifications, validation trials are required.

However, despite the promising available data on genome and transcriptome analysis in time and space, a new design of spatiotemporal patient-centric genomic and transcriptomic study with a specific prospective clinical protocol in nonmetastatic disease is required. Potential positive results from these trials will highlight the beginning of Precision Surgical Oncology.

Financial \& competing interests disclosure

The authors have no relevant affiliations or financial involvement with any organization or entity with a financial interest in or financial conflict with the subject matter or materials discussed in the manuscript. This includes employment, consultancies, honoraria, stock ownership or options, expert testimony, grants or patents received or pending, or royalties.

No writing assistance was utilized in the production of this manuscript. 


\section{References}

1. Siegel RL, Miller KD, Jemal A. Cancer statistics, 2019. CA Cancer J. Clin. 69(1), 7-34 (2019).

2. Shendure J, Ji H. Next-generation DNA sequencing. Nat. Biotechnol. 26(10), 1135-1145 (2008).

3. Elkon R, Agami R. Characterization of noncoding regulatory DNA in the human genome. Nat. Biotechnol. 35(8), 732-746 (2017).

4. Roukos DH. Spatiotemporal diversification of intrapatient genomic clones and early drug development concepts realize the roadmap of precision cancer medicine. Drug Discov. Today 22(8), 1148-1164 (2017).

5. Fellmann C, Gowen BG, Lin PC, Doudna JA, Corn JE. Cornerstones of CRISPR-Cas in drug discovery and therapy. Nat. Rev. Drug Discov. 16(2), 89-100 (2017).

6. Ben-David U, Beroukhim R, Golub TR. Genomic evolution of cancer models: perils and opportunities. Nat. Rev. Cancer 19(2), 97-109 (2019).

7. Lesterhuis WJ, Bosco A, Millward MJ, Small M, Nowak AK, Lake RA. Dynamic versus static biomarkers in cancer immune checkpoint blockade: unravelling complexity. Nat. Rev. Drug Discov. 16(4), 264-272 (2017).

8. Kyrochristos ID, Ziogas DE, Roukos DH. Dynamic genome and transcriptional network-based biomarkers and drugs: precision in breast cancer therapy. Med. Res. Rev. 39(3), 1205-1227 (2018).

9. Kyrochristos ID, Ziogas DE, Roukos DH. Drug resistance: origins, evolution and characterization of genomic clones and tumor ecosystem to optimize precise individualized therapy. Drug Discov. Today doi:10.1016/j.drudis.2019.04.008 (2019) (Epub ahead of print).

10. Ziogas DE, Kyrochristos ID, Roukos DH. Discovering novel valid biomarkers and drugs in patient-centric genomic trials: the new epoch of precision surgical oncology. Drug Discov. Today 23(11), 1848-1872 (2018).

11. Von Minckwitz G, Procter M, De Azambuja E et al. Adjuvant pertuzumab and trastuzumab in early HER2-positive breast cancer. $N$. Engl. J. Med. 377(2), 122-131 (2017).

12. Martin M, Holmes FA, Ejlertsen B et al. Neratinib after trastuzumab-based adjuvant therapy in HER2-positive breast cancer (ExteNET): 5-year analysis of a randomised, double-blind, placebo-controlled, Phase 3 trial. Lancet Oncol. 18(12), 1688-1700 (2017).

13. Primrose J, Falk S, Finch-Jones $\mathrm{M}$ et al. Systemic chemotherapy with or without cetuximab in patients with resectable colorectal liver metastasis: the new EPOC randomised controlled trial. Lancet Oncol. 15(6), 601-611 (2014).

14. Noh SH, Park SR, Yang HK et al. Adjuvant capecitabine plus oxaliplatin for gastric cancer after D2 gastrectomy (CLASSIC): 5-year follow-up of an open-label, randomised Phase 3 trial. Lancet Oncol. 15(12), 1389-1396 (2014).

15. Eggermont AM, Chiarion-Sileni V, Grob JJ et al. Prolonged survival in stage III melanoma with ipilimumab adjuvant therapy. N. Engl. J. Med. 375(19), 1845-1855 (2016).

16. Neoptolemos JP, Palmer DH, Ghaneh P et al. Comparison of adjuvant gemcitabine and capecitabine with gemcitabine monotherapy in patients with resected pancreatic cancer (ESPAC-4): a multicentre, open-label, randomised, Phase 3 trial. Lancet 389(10073), 1011-1024 (2017).

17. Pignon JP, Tribodet $\mathrm{H}$, Scagliotti GV et al. Lung adjuvant cisplatin evaluation: a pooled analysis by the LACE Collaborative Group. J. Clin. Oncol. 26(21), 3552-3559 (2008).

18. ENCODE Project Consortium. The ENCODE (ENCyclopedia Of DNA Elements) Project. Science 306(5696), 636-640 (2004).

19. Lawrence MS, Stojanov P, Mermel CH et al. Discovery and saturation analysis of cancer genes across 21 tumour types. Nature 505(7484), 495-501 (2014).

20. Eirew P, Steif A, Khattra J et al. Dynamics of genomic clones in breast cancer patient xenografts at single-cell resolution. Nature 518(7539), 422-426 (2015).

21. Murtaza M, Dawson SJ, Tsui DW et al. Non-invasive analysis of acquired resistance to cancer therapy by sequencing of plasma DNA. Nature 497(7447), 108-112 (2013).

22. Campbell JD, Alexandrov A, Kim J et al. Distinct patterns of somatic genome alterations in lung adenocarcinomas and squamous cell carcinomas. Nature Genet. 48(6), 607-616 (2016).

23. Nik-Zainal S, Davies H, Staaf J et al. Landscape of somatic mutations in 560 breast cancer whole-genome sequences. Nature 534(7605), 47-54 (2016).

24. Cancer Genome Atlas Research Network, Albert Einstein College Of Medicine, Analytical Biological Services et al. Integrated genomic and molecular characterization of cervical cancer. Nature 543(7645), 378-384 (2017).

25. Wang Z, Kim TB, Peng B et al. Sarcomatoid renal cell carcinoma has a distinct molecular pathogenesis, driver mutation profile, and transcriptional landscape. Clin. Cancer Res. 23(21), 6686-6696 (2017).

26. Bailey P, Chang DK, Nones K et al. Genomic analyses identify molecular subtypes of pancreatic cancer. Nature 531(7592), 47-52 (2016).

27. Dienstmann R, Vermeulen L, Guinney J, Kopetz S, Tejpar S, Tabernero J. Consensus molecular subtypes and the evolution of precision medicine in colorectal cancer. Nat. Rev. Cancer 17(4), 268 (2017). 
28. Cohen JD, Li L, Wang Y et al. Detection and localization of surgically resectable cancers with a multi-analyte blood test. Science 359(6378), 926-930 (2018).

29. Cohen JD, Javed AA, Thoburn C et al. Combined circulating tumor DNA and protein biomarker-based liquid biopsy for the earlier detection of pancreatic cancers. Proc. Natl Acad. Sci. USA 114(38), 10202-10207 (2017).

30. Clinical Trials Database: NCT02889978. https://clinicaltrials.gov/ct2/show/NCT02889978

31. Jamal-Hanjani M, Wilson GA, McGranahan N et al. Tracking the evolution of non-small-cell lung cancer. N. Engl. J. Med. 376(22), 2109-2121 (2017).

32. Desmedt C, Fumagalli D, Pietri E et al. Uncovering the genomic heterogeneity of multifocal breast cancer. Am. J. Pathol. 236(4), 457-466 (2015).

33. Yates LR, Gerstung M, Knappskog S et al. Subclonal diversification of primary breast cancer revealed by multiregion sequencing. Nat. Med. 21(7), 751-759 (2015).

34. Goswami RS, Patel KP, Singh RR et al. Hotspot mutation panel testing reveals clonal evolution in a study of 265 paired primary and metastatic tumors. Clin. Cancer Res. 21(11), 2644-2651 (2015).

35. Brastianos PK, Carter SL, Santagata S et al. Genomic characterization of brain metastases reveals branched evolution and potential therapeutic targets. Cancer Discov. 5(11), 1164-1177 (2015).

36. Joung JG, Oh BY, Hong HK et al. Tumor heterogeneity predicts metastatic potential in colorectal cancer. Clin. Cancer Res. 23(23), 7209-7216 (2017).

37. Frenel JS, Carreira S, Goodall J et al. Serial next-generation sequencing of circulating cell-free DNA evaluating tumor clone response to molecularly targeted drug administration. Clin. Cancer Res. 21(20), 4586-4596 (2015).

38. Tie J, Kinde I, Wang Y et al. Circulating tumor DNA as an early marker of therapeutic response in patients with metastatic colorectal cancer. Ann. Oncol. 26(8), 1715-1722 (2015).

39. Ueda M, Iguchi T, Masuda T et al. Somatic mutations in plasma cell-free DNA are diagnostic markers for esophageal squamous cell carcinoma recurrence. Oncotarget 7(38), 62280-62291 (2016). 